\title{
EDITORIAL
}

\section{New coronary imaging techniques: what to expect?}

\section{P J de Feyter, K Nieman}

Heart 2002;87:195-197

Coronary imaging is the ultimate challenge. During the last decade there have been great advances in this imaging technique, partly as a result of improved scanner hardware, but more because of advances in microprocessor technology

See end of article for authors' affiliations

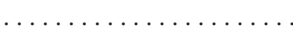

Correspondence to: PJ de Feyter, MD University Hospital Rotterdam, Thoraxcenter Bd 410, PO Box 2040, 3000 CA Rotterdam, The Netherlands; E-mail: defeyter@card.azr.nl

C diastole. images of the coronary lumen and plaque. plaque is stable, unstable or vulnerable?

\section{NON-INVASIVE CORONARY LUMEN IMAGING} oronary imaging is the ultimate challenge for any imaging technique. This is because the coronary arteries are small $(2-4 \mathrm{~mm}$ in diameter) and have a tortuous, complex, three dimensional course and are continuously in motion, except for a short period during mid

During the last decade we have witnessed great advances in cardiac imaging. This was partly as a result of improved scanner hardware, but more because of advances in microprocessor technology, which allow for rapid processing of extremely large quantities of data necessary for the acquisition, post-processing, and construction of hitherto unimaginable, non-invasively obtained

This raises two important questions. Firstly, has progress in non-invasive coronary imaging techniques gone so far that conventional invasive diagnostic coronary angiography has become redundant? Secondly, are these new imaging techniques capable of detecting which coronary

Magnetic resonance coronary angiography (MRCA), electron beam computed tomography (EBCT), and multi-slice computed tomography (MS-CT) have recently emerged as new noninvasive diagnostic techniques that possess the potential to replace conventional diagnostic angiography. ${ }^{1-4}$ The diagnostic accuracy to detect a significant coronary stenosis is presented in table 1. However, it should be noted that: firstly,

Table 1 Approximate diagnostic value of non-invasive coronary imaging techniques to detect significant coronary stenosis (> $50 \%$ lumen diameter stenosis)

\begin{tabular}{lccll}
\hline & EBCT & MS-CT & $\begin{array}{l}\text { MR-CA Free } \\
\text { breathing }\end{array}$ & $\begin{array}{l}\text { MR-CA } \\
\text { Breath-hold }\end{array}$ \\
\hline Assessability (\%) & $\sim 80$ & $\sim 70$ & $\sim 75$ & $\sim 70$ \\
Sensitivity (\%) & $\sim 80$ & $\sim 80$ & $\sim 50$ & $\sim 70$ \\
Specificity (\%) & $\sim 95$ & $\sim 95$ & $\sim 90$ & $\sim 95$ \\
Accuracy (\%) & $\sim 90$ & $\sim 90$ & $\sim 85$ & $\sim 90$ \\
\hline
\end{tabular}

EBCT, electron beam computed tomography; MS-CT, multi-slice computed tomography; MR-CA, magnetic resonance coronary angiography. the diagnostic value only concerns the detection of stenoses in the proximal and mid segment of the coronary tree, because the smaller distal segments cannot be adequately visualised; and secondly, only in $70-80 \%$ of these proximal and mid segments is image quality sufficient to allow a semiquantitative assessment.

At this point in time all three techniques have significant shortcomings (table 2), which all result in poor image quality and non-assessability of coronary vessel segments.

To replace diagnostic coronary angiography, coronary imaging requires a spatial resolution of $0.5 \times 0.5 \times 0.5 \mathrm{~mm}$ with a temporal resolution of 30-50 ms, while these images must be generated with a reliable and consistent image quality.

MR-CA, as it has been used in clinical practice, has a resolution of roughly $1.25 \times 1.25 \times 1.5 \mathrm{~mm}$ with a temporal resolution of $100-125 \mathrm{~ms}^{2}$ Higher resolution techniques are possible, but these require considerably longer acquisition time and thus greatly increase the likelihood of motion artefacts. Research concentrates on faster acquisition sequences (for instance True Fisp, VCATS), improvement of signal-to-noise ratio with higher magnetic fields ( 3 Tesla instead of 1-5 Tesla which is nowadays typical), and improvement of signal intensity with magnetic resonance imaging (MRI) contrast agents (currently tested in phase II trials).

EBCT has a spatial resolution of $0.7 \times 0.7 \times 1.5-$ $3 \mathrm{~mm}$ and a temporal resolution of $100 \mathrm{~ms}$. It is nowadays the fastest available acquisition technique. Technical refinements are expected to improve spatial and temporal (to as low as $50 \mathrm{~ms}$ ) resolution.

MS-CT angiography has a spatial resolution of $0.6 \times 0.6 \times 1.2 \mathrm{~mm}$ with a temporal resolution between $125-250 \mathrm{~ms}^{3}{ }^{4}$ Technical improvements,

Table 2 Shortcomings of non-invasive coronary imaging techniques

1. Insufficient spatial resolution (in particular $Z$ axis)

2. Inadequate temporal resolution

3. Rather long examination time (MRI)

4. Rather long breathhold (30-40 seconds)

5. Calcification obscures lumen (MS-CT, EBCT)

6. Considerable $x$ ray exposure (MS-CT, EBCT)

7. Lack of quantification

Abbreviations: $E B C T$, electron beam computed tomography; IVUS, intravascular ultrasound; MR-CA, magnetic resonance coronary angiography; MRI, magnetic resonance imaging; $M S-C T$, multi-slice computed tomography; $\mathrm{OCT}$, optical coherence tomography 
such as a larger number of thinner detector rows (to 16 rows), will increase spatial resolution (in particular $\mathrm{Z}$ axis resolution) and will allow faster data acquisition (to 20 second breathhold), whereas ECG triggered tube current modulation will reduce $x$ ray exposure. A further increase of the gantry rotation speed will also contribute to a better temporal resolution and shorter acquisition time.

Three dimensional image reconstruction allows postprocessing algorithms to construct coronary artery flythrough films, thereby creating virtual angioscopic imaging. ${ }^{5}$

In this issue an MS-CT coronary imaging data set was used to create "virtual coronaroscopy" which may evolve into a new method to assess complex coronary obstructions. ${ }^{6}$ Virtual angioscopy challenges any imaging technique and requires the highest resolution (ideally resulting in isotropic voxels) and high quality images to generate a reliable "scopy". At present, the quality of non-invasive coronary imaging with MS-CT, as well as with EBCT, allows virtual angioscopy, although the images are rather crude. ${ }^{56}$ Future studies will determine whether angioscopy offers diagnostic information additionally to existing two dimensional and three dimensional image representations.

\section{IDENTIFYING THE VULNERABLE PLAQUE}

Disruption (rupture or erosion) and intracoronary thrombosis of coronary plaques is a critical event, leading to an acute coronary syndrome, and healing often results in plaque growth.

It has become customary to classify coronary plaques: as stable with a low likelihood of disrupting; as vulnerable plaque with a high likelihood of rupture; or as unstable when it has actually ruptured.

The holy grail in coronary plaque imaging is a reliable in vivo method to discriminate between vulnerable and stable plaques. Several, invasive, catheter based techniques and non-invasive techniques are nowadays available to assess coronary plaque characteristics, which may provide clues concerning the stability or vulnerability of the plaque. The most important features of coronary plaques and related imaging modalities which are able to depict these features are presented in table 3.

The vulnerable plaque has a large lipid pool and a thin fibrous cap, which contains large numbers of macrophages, in particular at the shoulders of the plaque. Intracoronary angioscopy can establish the presence, but not the size, of a yellow lipid lesion and is therefore limited in assessing vulnerability. ${ }^{7}$ Intracoronary ultrasound can accurately provide the dimensions of the coronary plaque, but is not able to offer consistent detection of a lipid pool, although a hypoechogenetic zone within a plaque is highly suspicious for the presence of a lipid pool..$^{7-9}$. The thickness of the fibrous cap is critical and a cap with a thickness less than $150 \mu \mathrm{m}$ has a high likelihood of rupturing. ${ }^{10}$ The resolution of standard intracoronary ultrasound is inadequate to measure thin caps, but with a dedicated ultrasound acquisition technique it

Table 3 In vivo coronary plaque imaging

\begin{tabular}{ll}
\hline Pathology & Imaging modality \\
\hline Lipid pool (presence, size) & $2,3,5$ \\
Fibrous cap & 2 \\
Calcium & $6,2,1$ \\
Inflammation (temperature) & 4 \\
Remodelling & 2 \\
Intracoronary thrombus (disruption) & 3,1 \\
Endothelial dysfunction & 1 (acetylcholine challenge) \\
Luminal stenosis & $1,2,5,6$ \\
\hline
\end{tabular}

Coronary imaging techniques: 1 Diagnostic angiography; 2 Intracoronary ultrasound; 3 Intracoronary angioscopy; 4 Thermometry; 5 Magnetic resonance imaging, $6 X$ ray computed tomography (EBCT or MS-CT). appears possible to visualise thin rupture prone fibrous caps. ${ }^{11}$ Discrete rises in temperature at coronary plaques that have ruptured and led to acute coronary syndromes can be detected with recently developed catheters, ${ }^{12}$ and it appears that the presence of a temperature rise is a predictor of adverse cardiac events. ${ }^{13}$ This technique appears quite promising, but it needs to be combined with a visualisation technique to assess precisely the localisation of the vulnerable plaque within the coronary tree.

"Accurate detection of calcium in the coronary vessel wall, which is evidence of the presence of coronary atherosclerosis, can be achieved with EBCT and MS-CT"

Accurate detection of calcium in the coronary vessel wall, which is evidence of the presence of coronary atherosclerosis, can be achieved with EBCT and MS-CT. Calcium quantification may have prognostic value, particularly when adjusted for age, but whether this is additive to the conventional risk factors or Framingham score remains controversial. ${ }^{14}{ }^{15}$ The presence of calcification should not be interpreted as a sign of stability, nor can the absence of calcification exclude the presence of a coronary plaque, even of a vulnerable plaque, although the likelihood is low. ${ }^{14}$ It has recently been shown that, using intracoronary ultrasound: (1) the presence of an unstable plaque is positively associated with remodelling ${ }^{16} ;(2)$ large plaque area containing an echolucent zone is at increased risk of instability ${ }^{17}$; and (3) yellow plaques (detected with angioscopy) with increased distensibility and compensatory enlargement are predictive of a vulnerable plaque. ${ }^{18}$ However, the predictive value of each of these findings is too low to be clinically useful.

\section{EVOLVING NEW TECHNIQUES TO DETECT VULNERABLE PLAQUE}

Two catheter based techniques, Raman spectroscopy ${ }^{19}$ and optical coherence tomography (OCT), ${ }^{20}$ have the ability to distinguish between lipid plaques, fibrous plaques, and calcification; OCT is also able to measure fibrous cap thickness, as a result of its excellent spatial resolution (axial resolution 2-30 $\mu \mathrm{m}$ and lateral resolution 5-30 $\mu \mathrm{m}$ ). However, both techniques have significant problems, the most important being the absorbency of the signal by blood, which makes it difficult to use in human coronaries. Elastography is an ultrasound based technique that assesses the mechanical properties of components of the plaque on the basis of difference in hardness. ${ }^{21}$ The technique uses the radiofrequency data of a regular intravascular ultrasound (IVUS) system. In vitro studies have shown that elastography can discriminate between lipid rich and fibrous components ${ }^{21}$ and elastography is now being tested in the clinical setting.

Two dimensional, high resolution transthoracic echocardiography is able to measure left anterior descendens wall thickness and external diameter and may evolve as a technique to monitor subclinical coronary atherosclerosis. ${ }^{22}$

Plaque composition can be assessed by MS-CT on the basis of $x$ ray attenuation differences (Hounsfield units) of the various plaque components. The technique crudely allows investigators to distinguish between soft, intermediate, and calcific plaques, similar to the classification of plaques into soft, fibrous, and calcific using IVUS, to which these measurements were compared. ${ }^{23}$

In large arteries high resolution non-invasive MRI allowed detection of carotid and aortic atherosclerotic plaques, and the effect of lipid lowering on these plaques can be assessed using serial MRI studies. ${ }^{24}$

Preliminary data have shown that using high resolution MRI (in plane resolution $0.5-1.0 \mathrm{~mm}$, slice thickness 
3-5 mm), it was also possible to image non-invasively the coronary wall and the coronary plaque in a few patients. ${ }^{25} 26$

Serial in vivo MRI in atherosclerotic rabbits allowed the assessment of arterial remodelling, ${ }^{27}$ which is expected to be possible in the larger arteries in humans.

So far, radiotracer techniques to image coronary thrombi have not been successful enough to be used in clinical practice. But recently it was demonstrated that labelling a glycoprotein IIb/IIIa receptor inhibitor to technetium $99^{\mathrm{m}}$ allowed identification of an arterial thrombus in the left descending coronary artery in a canine model. ${ }^{28}$ This method is currently being tested in humans.

\section{STABILISATION OF THE PLAQUE}

Monitoring the efficacy of an intervention on progression of the plaque has until now exclusively been assessed with repeated angiography. Intracoronary ultrasound appears to be a more sensitive method in the detection of early plaque formation and changes in plaque volume. ${ }^{89}$ Change in the composition of the plaque can be induced by statin treatment ${ }^{29}$ and this process can be monitored by intracoronary ultrasound, because the plaque echogenicity changes. For example, a hypoechogenetic zone within a plaque (suggesting a lipid pool) may turn into a hyperechogenetic zone (suggesting fibrous tissue). ${ }^{30}$ However, although these echographically assessed changes in composition of the plaque are quite suggestive, this requires confirmation from histological studies which have not yet been performed.

\section{CONCLUSION}

The non-invasive coronary lumen imaging techniques MRCA, EBCT, and MS-CT are emerging as important techniques, but so far these techniques lack sufficient and consistent image quality to replace diagnostic coronary angiography. It may take another few years before these techniques have fully matured to make coronary angiography redundant. Various invasive and non-invasive coronary plaque imaging techniques are being developed and tested in the preclinical setting, but these are only in the early stages of research and development. Intracoronary ultrasound is currently the most advanced and clinically applicable tool to provide adequate information about coronary plaque dimensions and remodelling; however, this technique needs improvement in order to be able to assess vulnerability and stability of coronary plaques reliably. Intracoronary temperature measurement is feasible and clinical studies have just begun to assess its diagnostic and prognostic value.

\section{Authors' affiliations}

P J de Feyter, K Nieman, University Hospital Rotterdam, Thoraxcenter Bd 410, PO Box 2040, 3000 CA Rotterdam, The Netherlands

\section{REFERENCES}

1 Rensing BJ, Bongaerts AH, van Geuns RJ et al. Intravenous coronary angiography using electron beam computed tomography. Prog Cardiovasc Dis 1999;42:139-48

2 van Geuns RJ, Wielopolski PA, de Bruin HG, et al. Magnetic resonance imaging of the coronary arteries: techniques and results. Prog Cardiovasc Dis 1999:42:157-66.

3 Nieman K, Oudkerk M, Rensing BJ, et al. Coronary angiography with multi-slice computed tomography. Lancet 2001;357:599-603.

4 Achenbach S, Ulzheimer S, Baum U, et al. Noninvasive coronary angiography by retrospectively ECG-gated multislice spiral CT. Circulation 2000; 102:2823-8.

5 van Ooijen, P.M., Oudkerk M, van Geuns RJ, et al. Coronary artery fly-through using electron beam computed tomography. Circulation 2000;102:E6-10.
6 Schroeder S, Kopp AF, Ohnesorge B, et al. Virtual coronaroscopy using multi-slice computed tomography. Heart 2002;87:205-9.

7 de Feyter PJ, Ozaki Y, Baptista J, et al. Ischemia-related lesion characteristics in patients with stable or unstable angina. A study with intracoronary angioscopy and ultrasound. Circulation 1995;92:1408-13.

8 Nissen SE, Yock P. Intravascular ultrasound: novel pathophysiological insights and current clinical applications. Circulation 2001;103:604-16.

9 von Birgelen C, Slager CJ, Di Mario C, et al. Volumetric intracoronary ultrasound: a new maximum confidence approach for the quantitative assessment of progression-regression of atherosclerosis? Atherosclerosis 1995; 118(suppl):S103-13.

10 Loree HM, Kamm RD, Stringfellow RG, et al. Effects of fibrous cap thickness on peak circumferential stress in model atherosclerotic vessels. Circ Res 1992;71:850-8.

11 Hiro T, Fujii T, Yasumoto K, et al. Detection of fibrous cap in atherosclerotic plaque by intravascular ultrasound by use of color mapping of angle-dependent echo-intensity variation. Circulation 2001:103:1206-11.

12 Stefanadis C, Diamantopoulos L, Vlachopoulos C, et al. Thermal heterogeneity within human atherosclerotic coronary arteries detected in vivo: a new method of detection by application of a special thermography catheter. Circulation 1999;99:1965-71.

13 Stefanadis C, Toutouzas K, Tsiamis E, et al. Increased local temperature in human coronary atherosclerotic plaques: an independent predictor of clinical outcome in patients undergoing a percutaneous coronary intervention. J Am Coll Cardiol 2001:37:1277-83.

14 O'Rourke RA, Brundage BH, Froelicher VF, et al. American College of Cardiology/American Heart Association expert consensus document on electron-beam computed tomography for the diagnosis and prognosis of coronary artery disease. J Am Coll Cardiol 2000;36:326-40.

15 Taylor AJ, Burke AP, O'Malley PG, et al. A comparison of the Framingham risk index, coronary artery calcification, and culprit plaque morphology in sudden cardiac death. Circulation 2000;101:1243-8.

16 Schoenhagen P, Ziada KM, Kapadia SR, et al. Extent and direction of arterial remodeling in stable versus unstable coronary syndromes : an intravascular ultrasound study. Circulation 2000;101:598-603.

17 Yamagishi M, Terashima M, Awano K, et al. Morphology of vulnerable coronary plaque: insights from follow-up of patients examined by intravascular ultrasound before an acute coronary syndrome. J Am Coll Cardiol 2000;35: 106-11.

18 Takano M, Mizuno K, Okamatsu K, et al. Mechanical and structural characteristics of vulnerable plaques: analysis by coronary angioscopy and intravascular ultrasound. J Am Coll Cardiol 2001;38:99-104.

19 Romer TJ, Brennan JF 3rd, Fitzmaurice M, et al. Histopathology of human coronary atherosclerosis by quantifying its chemical composition with Raman spectroscopy. Circulation 1998;97:878-85.

20 Tearney GJ, Brezinski ME, Boppart SA, et al. Images in cardiovascular medicine. Catheter-based optical imaging of a human coronary artery. Circulation 1996;94:3013.

21 de Korte CL, Pasterkamp G, van der Steen AF, et al. Characterization of plaque components with intravascular ultrasound elastography in human femoral and coronary arteries in vitro. Circulation 2000:102:617-23.

22 Gradus-Pizlo I, Sawada SG, Wright D, et al. Detection of subclinical coronary atherosclerosis using two-dimensional, high-resolution transthoracic echocardiography. J Am Coll Cardiol 2001;37:1422-9.

23 Schroeder S, Kopp AF, Baumbach A, et al. Noninvasive detection and evaluation of atherosclerotic coronary plaques with multislice computed tomography. J Am Coll Cardiol 2001;37:1430-5

24 Corti R, Fayad ZA, Fuster V, et al. Effects of lipid-lowering by simvastatin on human atherosclerotic lesions: a longitudinal study by high-resolution, noninvasive magnetic resonance imaging. Circulation $2001 ; 104: 249-52$

25 Fayad ZA, Fuster V, Fallon JT, et al. Noninvasive in vivo human coronary artery lumen and wall imaging using black-blood magnetic resonance imaging. Circulation 2000;102:506-10.

26 Botnar RM, Stuber M, Kissinger KV, et al. Noninvasive coronary vessel wall and plaque imaging with magnetic resonance imaging. Circulation 2000;102:2582-7

27 Worthley SG, Helft G, Fuster V, et al. Serial in vivo MRI documents arterial remodeling in experimental atherosclerosis. Circulation 2000;101:586-9.

28 Mitchel J, Waters D, Lai T, et al. Identification of coronary thrombus with a llb/llla platelet inhibitor radiopharmaceutical, technetium- $99 \mathrm{~m}$ DMP-444: a canine model. Circulation 2000;101:1643-6.

29 Crisby M, Nordin-Fredriksson G, Shah PK, et al. Pravastatin treatment increases collagen content and decreases lipid content, inflammation, metalloproteinases, and cell death in human carotid plaques: implications for plaque stabilization. Circulation 2001;103:926-33.

30 Schartl M, Bocksch W, Koschyk DH, et al. Use of intravascular ultrasound to compare effects of different strategies of lipid-lowering therapy on plaque volume and composition in patients with coronary artery disease. Circulation 2001;104:387-92. 